\title{
Enhanced Power Factor of Higher Manganese Silicide via Melt Spin
}

\section{Synthesis Method}

\author{
Xiaoya $\mathrm{Shi}^{1}$, Xun $\mathrm{Shi}^{2,3}$, Yulong $\mathrm{Li}^{2}$, Ying $\mathrm{He}^{2}$, Lidong $\mathrm{Chen}^{2,3}$, and Qiang $\mathrm{Li}^{1, \mathrm{a})}$ \\ ${ }^{1}$ Condensed Matter Physics \& Materials Science Department, Brookhaven National Laboratory, \\ Upton, New York 11973, USA. \\ ${ }^{2}$ State Key Laboratory of High Performance Ceramics and Superfine Microstructure, Shanghai \\ Institute of Ceramics, Chinese Academy of Sciences, Shanghai 200050, China \\ ${ }^{3}$ CAS Key Laboratory of Materials for Energy Conversion, Shanghai Institute of Ceramics, \\ Chinese Academy of Sciences, Shanghai 200050, China
}

\section{Abstract}

We report on the thermoelectric properties of the Higher Manganese Silicide $\mathrm{MnSi}_{1.75}$ (HMS) synthesized by means of a one-step non-equilibrium method. The ultrahigh cooling rate generated from the melt-spin technique is found to be effective in reducing second phases, which are inevitable during the traditional solid state diffusion processes. Aside from being detrimental to thermoelectric properties, second phases skew the revealing of the intrinsic properties of this class of materials, for example the optimal level of carrier concentration. With this melt-spin sample, we are able to formulate a simple model based on a single parabolic band that can well describe the carrier concentration dependence of the Seebeck coefficient and power factor of the data reported in the literature. An optimal carrier concentration around $5 \times 10^{20} \mathrm{~cm}^{-3}$ at $300 \mathrm{~K}$ is predicted according to this model. The phase-pure melt-spin sample shows the largest power

\footnotetext{
a) Author to whom correspondence should be addressed. Electronic mail: liqiang@bnl.gov
} 
factor at high temperature, resulting in the highest $z T$ value among the three samples in this paper. And the maximum value is superior to those reported in the literatures.

\section{INTRODUCTION}

Higher manganese silicides (HMSs), with the formula $\mathrm{MnSi}_{1.75-x}$, exhibit a great potential for thermoelectric applications ( $p$-type) because of earth-abundant constituent elements, hightemperature oxidation resistance, environmental friendliness as well as a relatively large thermoelectric figure of merit, $z T$ (zT $~ 0.7$ around $800 \mathrm{~K}) .{ }^{[1-2]}$ HMSs are comprised of a series of different tetragonal phases, including $\mathrm{Mn}_{4} \mathrm{Si}_{7}, \mathrm{Mn}_{11} \mathrm{Si}_{19}, \mathrm{Mn}_{15} \mathrm{Si}_{26}$ and $\mathrm{Mn}_{27} \mathrm{Si}_{47},{ }^{[3-7]}$ which show comparable $a$ lattice parameter, and unusually different $c$ lattice parameters. It has a major challenge of obtaining single-phase HMS materials through traditional synthesis methods, such as direct solid state reaction and melt-water quenching methods due to the peritectic reaction and the slow diffusion rate of the Si atoms. ${ }^{[8-10]}$ The secondary phases of MnSi and Si would result in a degraded thermoelectric performance, as previously-reported. ${ }^{[11]}$ More importantly, second phases generally mask the intrinsic properties of this class of materials. One must minimize the presence of secondary phases in samples to gain better understanding of the nature of their electronic and phonon transport behavior, which is helpful for improving the thermoelectric performance of HMS based thermoelectric materials through chemical and structural tuning.

There are various methods reported of synthesizing HMS materials. ${ }^{[1,2,9,12-21]}$ In order to reduce the secondary phases, one of the most widely used approaches is the melting growth method followed by a quenching process, such as the arc-melting method. The secondary phases are effectively suppressed due to an increased cooling rate from the liquid phase to the solid phase 
during the quenching process. However, the cooling rate from water quenching of an arc melting method is not fast enough to suppress the phase separation into monosilicide and Si. It has always been a significant challenge to separate the intrinsic physical property of HMS from composites behavior because the second phases do affect the electrical and thermal properties. Thus, in order to study and further understand the electrical properties of this material, we developed a one-step melt-spin method (and proceeded directly from raw materials to HMS ribbons) in order to generate the ultrahigh cooling rates needed to obtain a virtually phase-pure sample (compared with traditional methods). A single parabolic band model was applied to analyze the data from both of our experiment and the literature. This model allows us to predict the optimal carrier concentration of HMS, which is a key parameter for further property improvement.

\section{EXPERIMENTAL PROCEDURE}

High-purity elements Mn (99.5\%, powder), Si (99.999\%, powder) were used as the starting materials. The powders were first prepared in the molar ratio $\mathrm{Mn}: \mathrm{Si}=1: 1.75$, and then mixed and cold-pressed into several disks (10 $\mathrm{mm}$ in diameter and $10 \mathrm{~mm}$ in thickness). For the meltspun sample (MS), the disks were embedded into a graphite crucible and then loaded into the quartz crucible with a $0.5 \mathrm{~mm}$ diameter nozzle, induction melted and ejected under a pressure of $0.02 \mathrm{MPa}$ of argon gas onto the high-speed rotating copper wheel with the linear speed of $38 \mathrm{~m} / \mathrm{s}$. The ribbon-shaped samples were collected and ground into fine powders. For the comparison samples, a set of the raw materials disks were placed into the carbon coated quartz tubes and sealed in vacuum. The solid state reaction sample disk (SSR) was slowly heated up to $1373 \mathrm{~K}$ and kept for 5 days, followed by directly shutting down the power of the furnace. Then the mixture 
was ground into powder and cold-pressed again and annealed at $1373 \mathrm{~K}$ for another 5 days. The third disk was melt-water quenching sample (MQ) which was first heated up to $1473 \mathrm{~K}$ and kept for 12 hours. Then the mixture was quenched into water. Both of the ingots from SSR and MQ processes were ground into fine powders. Finally, all the powders obtained from different synthesis methods were sintered using spark plasma sintering (SPS 2040, Simitomo) at $1123 \mathrm{~K}$ for 5 minutes under a 60MPa uniaxial pressure. The densities of the samples were measured by using the Archimedes' method and the result is listed in Table I.

All samples were examined by powder X-ray diffraction (XRD), with a Philips 3100E diffractometer ( $\mathrm{Cu}$ K $\alpha$ radiation, $\lambda=0.15418 \mathrm{~nm}, 40 \mathrm{kV} / 30 \mathrm{~mA}$ ). The low and high temperature thermoelectric properties were measured separately. The low temperature $(5 \mathrm{~K}$ to room temperature) data was collected with a Physical Property Measurement System (PPMS, Quantum Design). The high temperature measurements were carried out from room temperature to $850 \mathrm{~K}$. The thermal conductivity was measured via a laser flash technique (NETZSCH LFA427). The electrical conductivity, $\sigma$, was measured using a standard DC four-probe method. The Seebeck coefficient, $S$, was determined from the slope of the thermoelectromotive force, $\Delta E$, versus the temperature gradient $(0<\Delta T<4 \mathrm{~K})$. No anisotropy of the properties was observed during the measurements. The estimated measurement accuracies are listed below: $5 \%$ for electrical resistivity, $7 \%$ for Seebeck coefficient, $5 \%$ for thermal conductivity and $1 \%$ for density. The uncertainty of Hall measurement is $10 \%$. Thus the combined uncertainty for $z T$ was about $20 \%$. 


\section{RESULT AND DISCUSSION}

Figure 1 shows the XRD patterns of the HMS samples prepared via different synthesis methods, indicating all the samples are mainly comprised of HMS phase. The MS sample is virtually phase pure (below the x-ray machine's detection limit), while the MQ and SSR samples contain significant impurity phases - MnSi and $\mathrm{Si}$, as shown in Figure 1. No trace of secondary phases found in the XRD pattern indicates that the vastly increased cooling rate is beneficial for eliminating the secondary phases. In contrast, the SSR sample contains large amounts of secondary phases. This is probably due to an incomplete reaction during the solid state reaction process. Further, materials density provides another piece of information that is crucial for assessing the sample quality. We found the density of the MS sample is almost at the theoretical value (>99\%), which is much higher than those of the MQ (95.2 \%) and the SSR (93.4 \%) samples, as listed in Table I below. As a matter of fact, the secondary phases are unfavorable for sintering and the Si elementary substance tends to lower the density appreciably.

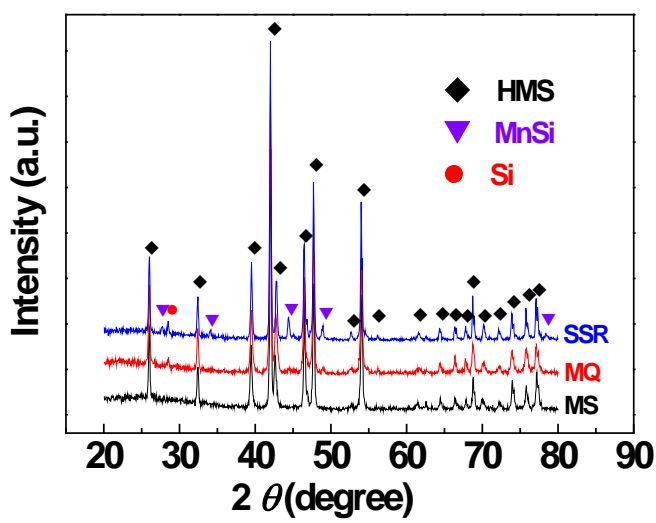

FIG. 1. Powder X-ray diffraction patterns of $\mathrm{MnSi}_{1.75}$ samples using different synthesis methods.

The symbols $\bullet$ show the peak of the HMS phase while the symbols $\boldsymbol{\nabla}$ and $\bullet$ represent the second phases $\mathrm{MnSi}$ and $\mathrm{Si}$, respectively. MS, MQ and SSR represent melt-spin, melt-water 
quenching and solid state reaction samples, respectively.

The low-temperature electrical properties are summarized in Figure 2. The electrical resistivity increases with increasing temperature, indicating metallic conduction behavior. The MS sample shows the lowest resistivity among three samples within the whole temperature range. This can be attributed to the fact that it has the largest carrier concentration and also a much higher density. Here, we used the Hall carrier concentration, $p_{H}$, instead of the chemical carrier concentration, $p$, because of similar Seebeck coefficients, indicating very close electrochemical potentials for the three samples. The chemical carrier concentration and the Hall carrier concentration could be related via electrochemical potential through the expressions below: ${ }^{[22,23]}$ $p=r_{H} \cdot p_{H}$,

where $r_{H}=\frac{3(1 / 2+2 \lambda) F_{2 \lambda-1 / 2}(\eta)}{2(1+\lambda)^{2} F_{\lambda}^{2}(\eta)} \cdot F_{1 / 2}(\eta)$,

with the Fermi integrals $F_{j}(\eta)$ defined by

$$
F_{j}(\eta)=\int_{0}^{\infty} \frac{\xi^{j} d \xi}{1+\exp (\xi-\eta)}
$$

Here, $\lambda$ is the scattering parameter, which is related to the energy dependence of the carrier relaxation time, $\tau$, such that $\tau=\tau_{0} \cdot \varepsilon^{\lambda-1 / 2}$ where the expression for $\tau_{0}$ depends on $\lambda$. $\xi$ is the reduced carrier energy, and $\eta$ represents the reduced electrochemical potential. The value of $\eta$ could be obtained via analysis of the Seebeck coefficient, $S$, data through the equation below,

$$
S=-\frac{k_{B}}{e}\left(\frac{(2+\lambda) F_{\lambda+1}(\eta)}{(1+\lambda) F_{\lambda}(\eta)}-\eta\right),
$$

Here, $k_{B}$ and $e$ are the Boltzmann constant and the electronic charge, respectively. By assuming that the acoustic phonon scattering (the scattering mechanism will be discussed later and acoustic 
phonon scattering makes $\lambda=0)$, ${ }^{[22,23]}$ the $r_{H}$ values can be calculated (listed in Table I). The Hall carrier concentration was calculated through $p_{H} \cdot e=1 / R_{H}$ by measuring the Hall coefficient $R_{H}$. The $r_{H}$ values at room temperature are slightly above 1.0 so that it is reasonable to take the Hall carrier concentration as the chemical carrier concentration in this paper directly. Thus, the chemical carrier concentration of the MS sample should be $1.34 \times 10^{21} \mathrm{~cm}^{-3}$, which is a quite high value compared with that of the state-of-the-art thermoelectric materials.
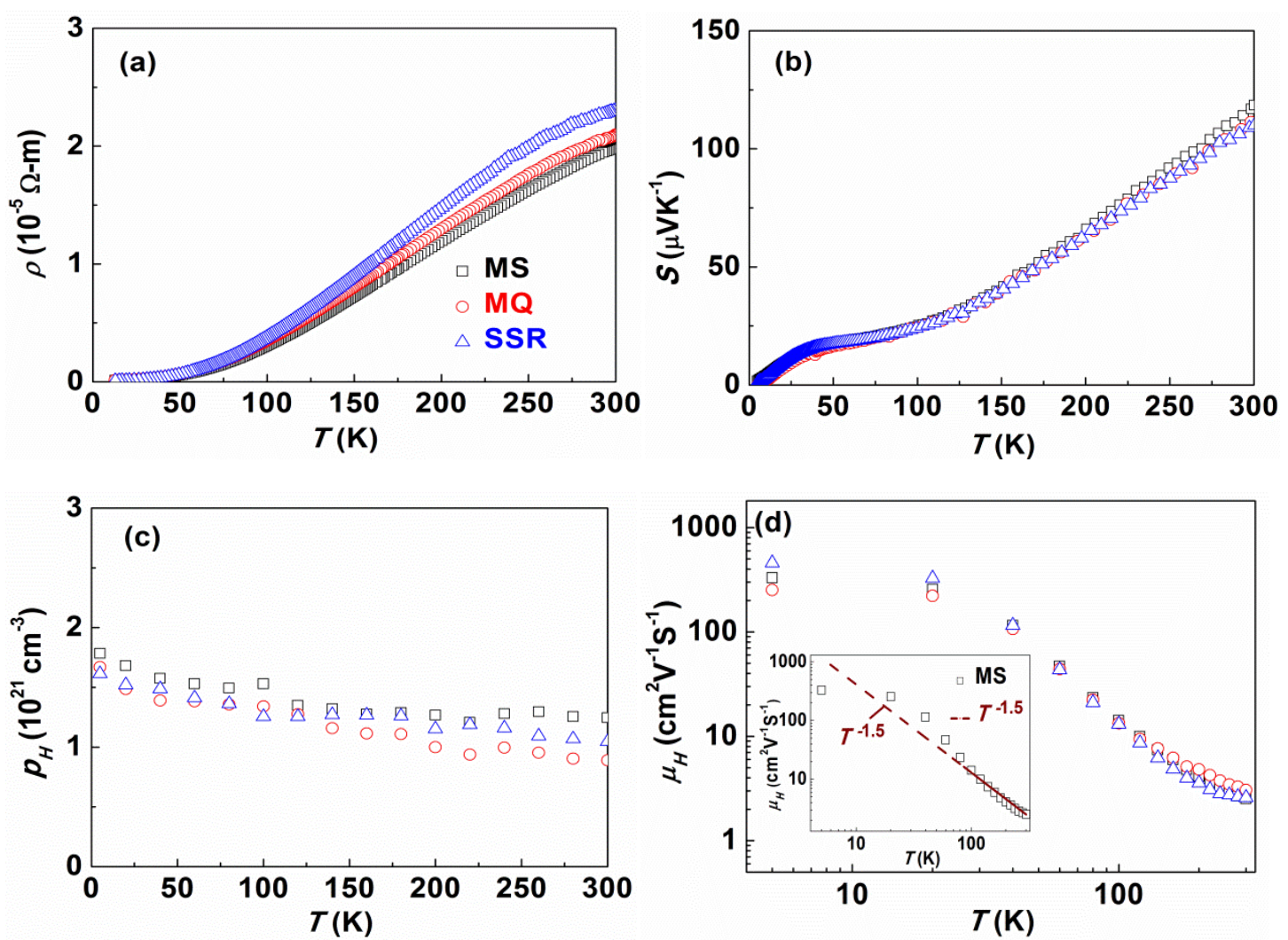

FIG. 2. Temperature dependence of (a) electrical resistivity, $\rho$, (b) Seebeck coefficient, $S$, (c) Hall carrier concentration, $p_{H}$ and (d) Hall mobility, $\mu_{H}$, for all the samples from $5 \mathrm{~K}$ to $300 \mathrm{~K}$. The insert figure in (d) represents MS sample by comparing with the power law of $T^{1.5}$ (dashed line).

By combining the resistivity and Hall carrier concentration data, we obtained the temperature dependence of the Hall mobility, as shown in Figure 2d. The three samples exhibit very similar 
mobility value and temperature dependence. MS data was emphasized in the insert figure separately in order to investigate the scattering mechanism. It is clear to see that the experimental data follows the power law of $T^{1.5}$ (the dashed line in the insert figure) quite well, indicating that the acoustic phonon scattering dominates in the MS sample at high temperature. The acoustic phonon scattering mechanism makes $\lambda=0$, as we mentioned above, and equation (4) can be simplified to: $:^{[22,23]}$

$$
S=-\frac{k_{B}}{e}\left(\frac{2 F_{1}(\eta)}{F_{0}(\eta)}-\eta\right)
$$

According to the Boltzmann transport equations (within the single parabolic band assumption), the Hall carrier concentration $p_{H}$ is given by:

$$
p_{H}=\frac{4 \pi\left(2 m^{*} k_{B} T\right)^{3 / 2}}{h^{3}} F_{1 / 2}(\eta)
$$

where $h$ and $m^{*}$ stand for Planck constant and the effective mass, respectively. From equations (5) and (6) we obtain the $S$ versus $p_{H}$ relationship under a single parabolic band model (SPB) by assuming acoustic phonon scattering and only one carrier type in the system. The data from the literature was also plotted in Figure 3 in order to make a comparison. It is interesting to note that the single-phase HMS from Ref. 17 fits the SPB line quite well and so do the Ge-doped samples from Ref. 19 and Al-doped samples from Ref. 21. We also notice that another batch of Al-doped samples from Ref. 16, which were also prepared via the melt-spin method, follow the theoretical line when the Al doping levels are low (the higher carrier concentration dots). However, the data starts to deviate from the SPB line at higher Al content (lower carrier concentration dots), properly due to the extremely low solubility of the $\mathrm{Al}$ atoms in the system as reported. ${ }^{[16,21]}$ And the formation of secondary phases may result in a reduced Seebeck in some cases. In addition, our 
MQ and SSR comparison samples are far below the theoretical line, which demonstrates again that the secondary phases are detrimental for the electrical properties.

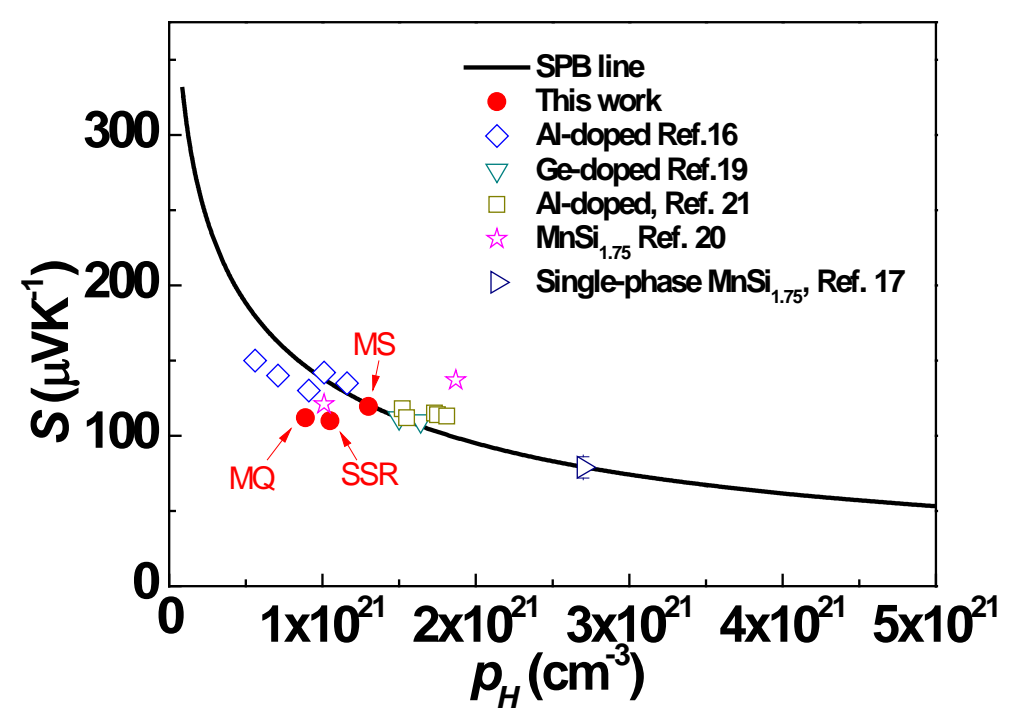

FIG. 3. Hall carrier concentration dependence of Seebeck coefficient at room temperature. The solid line is generated from single parabolic band model (SPB) based on the MS sample's data. All the other hollow dots are from the literatures.

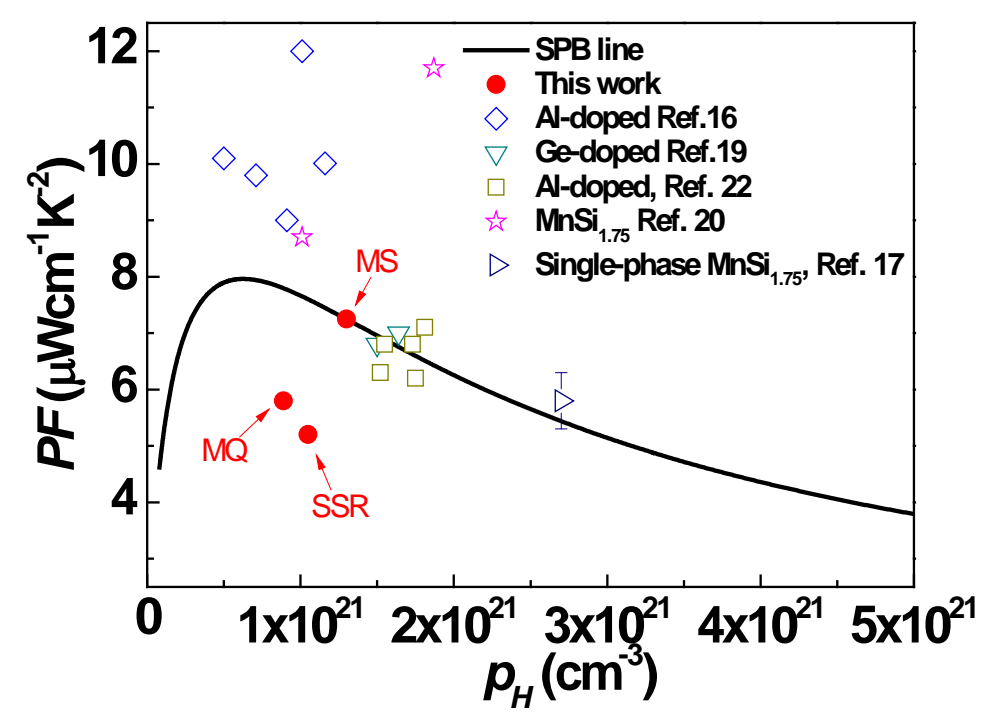

FIG. 4. Hall carrier concentration dependence of power factor $P F$ at room temperature. The calculated line is generated from single parabolic band model (SPB) based on the MS sample's 
data.

Table 1. Room temperature electrical performance of HMS samples.

\begin{tabular}{cccc}
\hline Properties & MS & MQ & SSR \\
\hline Density & $99.5 \%$ & $95.2 \%$ & $93.4 \%$ \\
$\rho\left(10^{-5} \Omega-\mathrm{m}\right)$ & 1.95 & 2.14 & 2.30 \\
$\left.S(\mu \mathrm{VK})^{-1}\right)$ & 119 & 112 & 110 \\
Power factor $\left(\mu \mathrm{Wcm}^{-1} \mathrm{~K}^{-2}\right)$ & 7.3 & 5.9 & 5.3 \\
$p_{H}\left(10^{21} \mathrm{~cm}^{-3}\right)$ & 1.24 & 0.89 & 1.04 \\
$r_{H}$ & 1.081 & 1.076 & 1.075 \\
$\mu_{H}\left(\mathrm{~cm} \mathrm{~V}^{-1} \mathrm{~S}^{-1}\right)$ & 2.5 & 3.1 & 2.6 \\
Effective mass $\left(m^{*} / m_{e}\right)$ & 7.4 & 5.5 & 6.0 \\
\hline
\end{tabular}

The theoretical value of the power factor $(P F)$ can be calculated under the SPB model to predict the electrical performance as a function of Hall carrier concentration. Here, we again use the data from the MS sample to generate the theoretical curve and the PF could be expressed based on the following equations: ${ }^{[23,24]}$

$P F=S^{2} \sigma$

$\sigma=p_{H} e \mu_{H}$

$\mu_{H}=\frac{\mu_{0} \pi^{1 / 2} F_{\lambda}(\eta)}{2 \Gamma(1+\lambda) F_{1 / 2}(\eta)}$.

Here, $\mu_{0}$ is the intrinsic mobility, related to the relaxation time $\tau_{0}$ by $\mu_{0}=e \tau_{0} / m^{*}{ }^{[23]} P F$ and $\eta$ is finally translated to $p_{H}$ via Eq. 5. Figure 4 shows the calculated Hall carrier concentration dependence of $P F$ at $300 \mathrm{~K}$. Again, the data of Ge-doped samples from Ref. 19 and the data from the single crystal from Ref.17 fall on the theoretical curve very well. In addition, the $P F$ values of 
Al-doped samples from Ref. 21 are also found to be clustered around the theoretical line. However, Al-doped samples from Ref. 16 are rather unusual, exhibiting much higher $P F$ than expected, due to the extremely low electrical resistivity even though the Seebeck is lower than theoretical expectation. This behavior might be associated with the impurities in those samples. An optimal carrier concentration $p^{*}$ of approximately $5 \times 10^{20} \mathrm{~cm}^{-3}$ is obtained as shown in Figure 4. Furthermore, we can estimate that $p^{*}$ for the $z T$ value is also around $5 \times 10^{20} \mathrm{~cm}^{-3}$ because the thermal conductivity does not change much under different carrier concentrations according to the previous studies on the HMS. One can estimate the high temperature $p^{*}$ from room temperature value according to the rough relationship $p^{*} \propto\left(m^{*} T\right)^{3 / 2}$ proposed by Ioffe ${ }^{[25]}$ under the assumption that only one type charge carrier contributes to the transport. If we consider the effective mass $m^{*}$ as a temperature-independent constant, the $p^{*}$ should increase with increasing temperature. A crude estimation of the $p^{*}$ at $600 \mathrm{~K}$, which is well below the bipolar region of HMS system, is around $1.5 \times 10^{21} \mathrm{~cm}^{-3}$. This value is comparable to the MS carrier concentration at room temperature. Due to the nearly flat carrier concentration of MS sample near room temperature region, it is reasonable to consider the temperature-independent behavior of carrier concentration at higher temperature region (above $300 \mathrm{~K}$ ). This is also supported by the linearly enhanced Seebeck data below $600 \mathrm{~K}$ shown in Figure 5a. As a result, it is expected that our MS sample may reach the optimal electrical performance in a certain temperature region around $600 \mathrm{~K}$. In principle, the higher the temperature, the larger the $p^{*}$ value, if we ignore the bipolar behavior at high temperatures. In fact, an optimal carrier concentration of $p^{*} 2 \times 10^{21} \mathrm{~cm}^{-3}$ at $832 \mathrm{~K}$ has been reported (Ref. 21). Based on the Ioffe's law, this optimal value agrees well with what we predicted from the data at room temperature in Figure 4. 

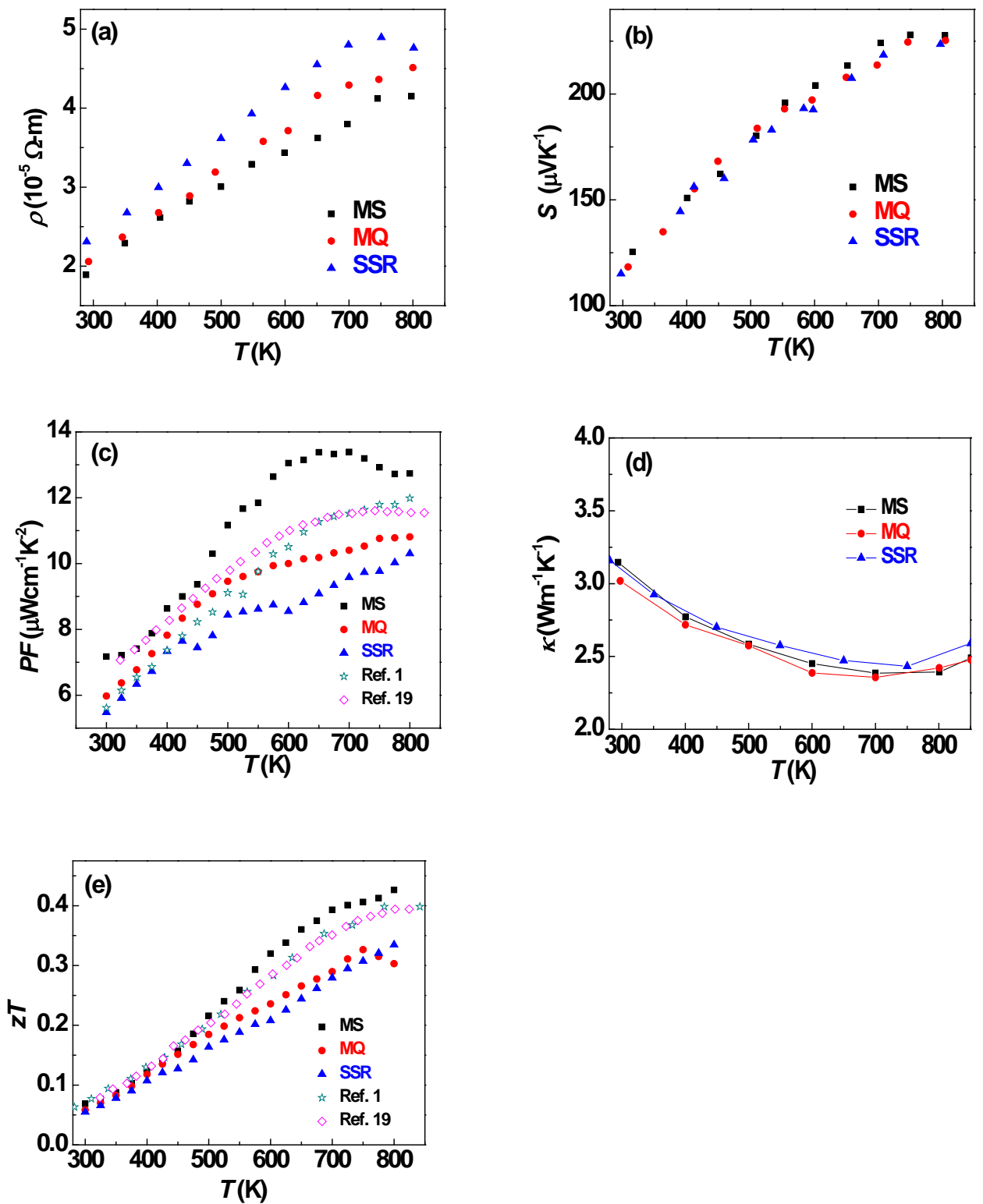

FIG. 5. Temperature dependence of (a) electrical resistivity, $\sigma$, (b) Seebeck coefficient, $S$, (c) power factor, $P F$, (d) thermal conductivity, $\kappa$ and (e) figure of merit $z T$ of MS, MQ and SSR samples. The power factor and figure of merit zT from ref. 1 and ref. 19 are also plotted in FIG.

5c and FIG. 5e for comparison.

Figure 5 shows the high temperature thermoelectric properties of the MS, MQ and SSR samples. The MS sample shows the lowest electrical resistivity among the three samples. This is because 
that the secondary phases MnSi and Si affect the electrical resistivity not only by producing carrier scattering centers but also by lowering the density of the material. These secondary phases are detrimental to the electrical performance. The similar behavior has been reported in the recent study on the high purity HMS grown by Chemical Vapor Transport method. ${ }^{[26]}$ A peak value of the Seebeck coefficient can be observed around $750 \mathrm{~K}$, which corresponds to the onset of the up-turn of the thermal conductivity, attributed to the bipolar behavior at high temperatures. The band gap of the system can be estimated from the maximum value of the Seebeck coefficient according to the equation: ${ }^{[27]}$

$E_{\mathrm{g}}=2 e S_{\max } T$

A band gap of $0.35 \mathrm{eV}$ is obtained, a reasonable value consistent with the previous report. ${ }^{[28]}$

As shown in Figure 5c, a much higher power factor can be achieved in the high temperature region for the MS sample, especially near $600 \mathrm{~K}$, where an optimal carrier concentration is expected to be reached in our MS sample. The temperature dependence of thermal conductivity, which is plotted in Figure 5d, does not vary much under different concentrations of secondary phases. This result again is consistent with what has been reported in the previous studies. ${ }^{[21,26]}$ The high-temperature (above room temperature) figure of merit $z T$ was calculated and presented in Figure 5e. Due to the increased electrical performance, the improved $z T$ was observed above $500 \mathrm{~K}$, which is the optimal temperature region as mentioned above. The $z T$ value of undoped HMS reaches 0.42 at $800 \mathrm{~K}$ in the melt spin sample, which is higher than that of other samples made by traditional methods. And it is quite promising to further improve the thermoelectric property of the HMS system by decreasing the thermal conductivity through alloying or doping approaches. We suggest future work should be focused on increasing the band-gap through 
doping methods in order to eliminate the bipolar effect or move the bipolar point beyond the temperature region of interest and at the same time, to tune the carrier concentration to an optimal level.

\section{CONCLUSION}

Higher manganese silicides were synthesized through a one-step melt-spin process, as well as by other traditional processes of solid state reaction and melting followed by water quenching methods, respectively. The MS sample shows pure HMS phase while MQ and SSR samples both contain secondary phases, $\mathrm{MnSi}$ and $\mathrm{Si}$, according to XRD results. The secondary phases are detrimental to the electrical performance by lowering the density and the carrier mobility of the material. However, the thermal transport of HMS is not sensitive to the concentration of secondary phases. By introducing the single parabolic band model, the optimal carrier concentration of HMS at room temperature was predicted to be $5 \times 10^{20} \mathrm{~cm}^{-3}$. Based on this simple model, we calculated the optimal carrier concentrations at different temperatures, and that can well describe the general behavior of this class of materials. In addition, we show that melt-spinning technique is a superior method for obtaining high quality manganese silicide samples with better electrical performance.

\section{Acknowledgement}

This work was supported by the US Department of Energy, Office of Basic Energy Science, Materials Sciences and Engineering Division, under contract no. DEAC0298CH10886. A portion of this work was performed at Shanghai Institute of Ceramics, Chinese Academy of Sciences (SICCAS). SICCAS is supported by National Natural Science Foundation of China (NSFC) under 
the No. 51121064 and 51222209. We also thank Dr. Ivo K. Dimitrov for critical reading of the manuscript and useful suggestions.

\section{References}

${ }^{1}$ V.K. Zaitsev, Thermoelectrics Handbook, Chap. 25, ed. D.M. Rowe (Boca Raton: CRC Press, 1995).

${ }^{2}$ M.I. Fedorov and V.K. Zaitsev, Thermoelectrics Handbook, ed. D.M. Rowe (Boca Raton: CRC Press, 2006), p. 3.

${ }^{3}$ U. Gottlieb, A. Sulpice, B. Lambert-Andron, and O. Laborde, J. Alloys Compd. 361, 13 (2003).

${ }^{4}$ O. Schwomma, H. Nowotny, and A. Wittman, Monatsch. Chem. 94, 681 (1963).

${ }^{5}$ O. Schwomma, A. Preisinger, H. Nowotny, and A. Wittman, Monatsch. Chem. 95, 1527 (1964).

${ }^{6}$ H.W. Knott, M.H. Mueller, and L. Heaton, Acta Crystallogr. 23, 549 (1967).

${ }^{7}$ G. Zwilling and H. Nowotny, Monatsch. Chem. 104, 668 (1973).

${ }^{8}$ E. Groß, M. Riffel, and U. Stöhrer, J Mater Res, 10, 34 (1995).

${ }^{9}$ I.Aoyama, H.Kaibe,L.Rauscher, T.Kanda, M.Mukoujima, S. Sano, and T. Tsuji, Jpn. J. Appl. Phys., 44, 4275 (2005).

${ }^{10}$ I. Kawasumi, M. Sakata, I. Nishida, and K. Masumoto, J. Mater. Sci. 16, 355 (1981).

${ }^{11}$ Umemoto M, Liu Z, Omatsuzawa R, Tsuchiya K. Meter Sci Forum, 343-3, 918 (2000).

${ }^{12}$ I. Nishida, K. Masumoto, I. Kawasumi, and M. Sakata, J. Less-Common Met. 71, 293 (1980).

${ }^{13}$ T. Kojima, I. Nishida, and T. Sakata, J. Cryst. Growth, 47, 589 (1979).

${ }^{14}$ I. Aoyama, M.I. Fedorov, V.K. Zaitsev, F.Y. Solomkin, I.S. Eremin, A.Y. Samunin, M. Mukoujima, S. Sano, and T. Tsuji, Jpn. J. Appl. Phys. 44, 8562 (2005).

${ }^{15}$ A.J. Zhou, T.J. Zhu, H.L. Ni, Q. Zhang, and X.B. Zhao, J. Alloys Compd. 455, 255 (2008).

${ }^{16}$ W. H. Luo, H. Li, F. Fu, W. Hao, and X. F. Tang, J. Electron. Mater. 40, 1233 (2011). 
${ }^{17}$ H. Udono, K. Nakamori, Y. Takahashi, Y. Ujiie, I. J. Ohsugi, and T. Iida, J. Electron. Mater. 40, 1165 (2011).

${ }^{18}$ W. H. Luo, H. Li, Y. G. Yan, Z. B. Lin, X. F. Tang, Q. J. Zhang, and C. Uher, Intermetallics, 19, 404 (2011).

${ }^{19}$ A. J. Zhou, T. J. Zhu, X. B. Zhao, S. H. Yang, T. Dasgupta, C. Stiewe, R. Hassdorf, and E. Mueller, J. Electron. Mater. 39, 2002 (2010).

${ }^{20}$ G. Liu, Q. M. Lu, X. Zhang, J. X. Zhang, Y, and J, Shi, J. Electron. Mater. 41, 1450 (2012).

${ }^{21}$ X. Chen, A. Weathers, D. Salta, L. B. Zhang, J. S. Zhou, J. B. Goodenough, and L. Shi, J. Appl. Phys., 114, 173705 (2013).

${ }^{22}$ V. I. Fistul, Heavily Doped Semiconductors (Plenum Press, NewYork, 1969).

${ }^{23}$ A. F. May, E. S. Toberer, A. Saramat, and G. J. Snyder, Phys. Rev. B, 80, 12505 (2009).

${ }^{24}$ E. S. Toberer, A. F. May, B. C. Melot, E. Flage-Larsen, and G. J. Snyder, Dalton Transactions, 39, 1046 (2010).

${ }^{25}$ A. F. Ioffe, Semiconductor thermoelements, and Thermoelectric cooling , Infosearch , London 1957 .

${ }^{26}$ S. N. Girard, X. Chen, F. Meng, A. Pokhrel, J. S. Zhou, L. Shi and S. Jin, Chem. Mater. 26, 5097-5104 (2014).

${ }^{27}$ H. J. Goldsmid and J. W. Sharp, J. Electron. Mater. 28, 869, (1999).

${ }^{28}$ D.B. Migas, V.L. Shaposhnikov, A.B. Filonov, V.E. Borisenko, and N.N. Dorozhkin, Phys. Rev. B 77, 075205 (2008). 